\title{
Left Ventricular Hypertrophy in a Patient with AL Amyloidosis: A Rare Overlap of Two Cardiomyopathies
}

\author{
Brock $M^{1}$, Sanchorawala $V^{2,3}$, Berk $\mathrm{JL}^{3}$, Cui $\mathrm{H}^{3,4}$, Gopal $\mathrm{DM}^{3,5}, \mathrm{O}^{\prime}$ Hara $\mathrm{C}^{3,4}$, Ruberg $\mathrm{FL}^{3,5,6}$ and Siddiqi \\ $O K^{3,5^{*}}$
}

${ }^{1}$ Department of Internal Medicine, Boston Medical Center, USA

${ }^{2}$ Division of Hematology and Oncology, Boston University School of Medicine, Boston Medical Center, USA

${ }^{3}$ Amyloidosis Center, Boston University School of Medicine, USA

${ }^{4}$ Department of Pathology, Boston University School of Medicine, Boston Medical Center, USA

${ }^{5}$ Section of Cardiovascular Medicine, Department of Medicine, Boston University School of Medicine, Boston Medical Center, USA

${ }^{6}$ Department of Radiology, Boston University School of Medicine, Boston Medical Center, USA

*Corresponding author: Omar K Siddiqi, MD, Section of Cardiovascular Medicine, Boston Medical Center, 88 East Newton Street, Boston, MA 02118, USA, Tel: 617-638-8771, Fax: 617-638-8756

\begin{abstract}
A 62-year-old woman with newly diagnosed systemic AL amyloidosis was evaluated for severely increased left ventricular (LV) wall thickness. Her echocardiographic, cardiac $\mathrm{MRI}$, and genetic findings led to a diagnosis of hypertrophic cardiomyopathy (HCM). Five years after achieving relative a partial hematologic response with chemotherapy, the patient died after developing rapidly progressive heart failure and cardiogenic shock. A full autopsy diagnosed both cardiac amyloidosis and HCM. The overlapping phenotypes of these two diseases resulted in a clinical diagnostic dilemma. This case highlights the importance of tissue diagnosis and genetic analysis in the evaluation of LV wall thickening when conflicting clinical features suggest divergent diagnoses.
\end{abstract}

\section{Keywords}

AL amyloidosis, Transthyretin, Hypertrophic cardiomyopathy

\section{Introduction}

We present a case of increased left ventricular wall thickness in a patient with $\mathrm{AL}$ amyloidosis. The patient was ultimately diagnosed with both hypertrophic cardiomyopathy and AL cardiac amyloidosis. The overlapping phenotypes of these two diseases resulted in a clinical diagnostic dilemma. This case highlights the importance of tissue diagnosis and genetic analysis in the evaluation of LV wall thickening when conflicting clinical features suggest divergent diagnoses. In addition, clinicians should consider superimposition of $A L$ amyloid cardiomyopathy in patient with rapid clinical deterioration.

\section{Case Description}

A 62-year-old woman with hypertension and newly diagnosed $A L$ amyloidosis was initially referred to the Boston University/Boston Medical Center Amyloidosis Center in 2006 for evaluation of suspected amyloidosis and increased left ventricular wall thickness noted by echocardiogram. There was no significant family history of heart disease or sudden cardiac death. A bladder biopsy obtained during evaluation for persistent hematuria revealed extensive amyloid disposition. A bone marrow biopsy xsmarrow biopsy. This was accompanied by markedly elevated serum lambda free light chains and monoclonal lambda protein in urine and serum immuno fixation electrophoresis, confirming the diagnosis of systemic AL amyloidosis (Table 1). Treatment with nine cycles of melphalan and dexamethasone resulted in a very good partial hematologic response with normaliza-

Citation: Brock M, Sanchorawala V, Berk JL, Cui H, Gopal DM, et al. (2019) Left Ventricular Hypertrophy in a Patient with AL Amyloidosis: A Rare Overlap of Two Cardiomyopathies. Int Arch Cardiovasc Dis 3:020. doi.org/10.23937/2643-3966/1710020

Accepted: August 24, 2019; Published: August 26, 2019

Copyright: (C) 2019 Brock M, et al. This is an open-access article distributed under the terms of the Creative Commons Attribution License, which permits unrestricted use, distribution, and reproduction in any medium, provided the original author and source are credited. 
Table 1: Hematological workup resulting in diagnosis of plasma cell dyscrasia.

\begin{tabular}{|l|l|l|}
\hline Test & Value & Normal \\
\hline IgG & $1150 \mathrm{mg} / \mathrm{dL}$ & $700-1600$ \\
\hline IgA & $166 \mathrm{mg} / \mathrm{dL}$ & $70-1600$ \\
\hline IgM & $130 \mathrm{mg} / \mathrm{dL}$ & $46-304$ \\
\hline Kappa FLC & $11.5 \mathrm{mg} / \mathrm{L}$ & $3.3-19.4$ \\
\hline Lamda FLC & $155.0 \mathrm{mg} / \mathrm{L}$ & $5.7-26.3$ \\
\hline FLC ratio & 0.07 & $0.26-1.65$ \\
\hline dFLC & $143.5 \mathrm{mg} / \mathrm{L}$ & $6.0-8.2$ \\
\hline Total protein & $7.1 \mathrm{~g} / \mathrm{dL}$ & $3.35-5.40$ \\
\hline Serum albumin & $4.27 \mathrm{~g} / \mathrm{dL}$ & $0.5-1.1$ \\
\hline Serum creatinine & $0.9 \mathrm{mg} / \mathrm{dL}$ & \\
\hline SIFE & Monoclonal lgG lambda gammopathy & \\
\hline UIFE & Monoclonal lgG lambda gammopathy & $0-150$ \\
\hline Urine protein excretion & $207 \mathrm{mg} / 24 \mathrm{hr}$ & \\
\hline HCM cardiac sarcomere mutations & MYH7 variant & \\
\hline Bone Marrow Biopsy & $5 \%$ CD138 positive plasma cells with lambda light chain staining with & \\
\hline
\end{tabular}

FLC: Free Light Chains; HCM: Hypertrophic Cardiomyopathy; SIFE: Serum Immunofluorescence Electrophoresis; UIFE: Urine Immunofluorescence Electrophoresis; dFLC: Difference in involved and uninvolved free light chain levels; IHC: Immunohistochemistry.

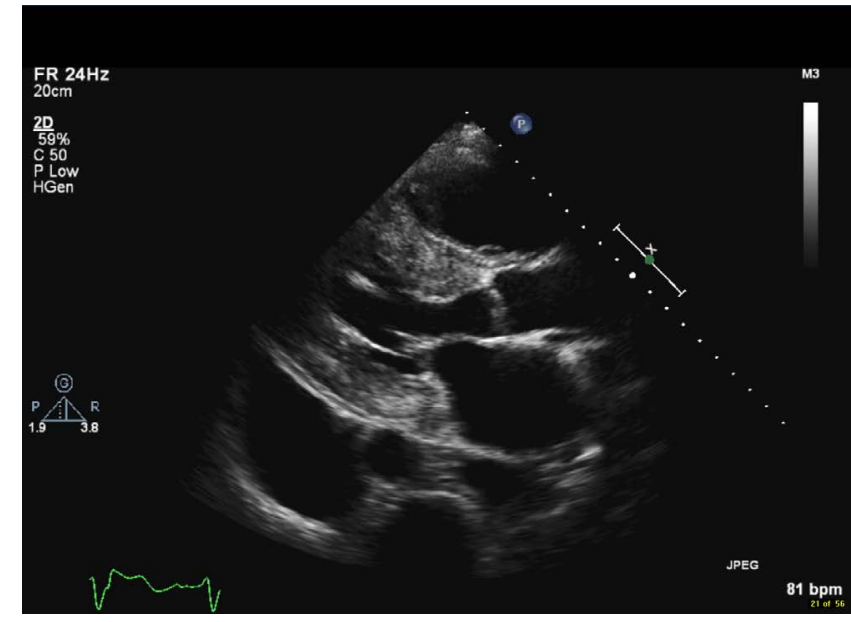

A

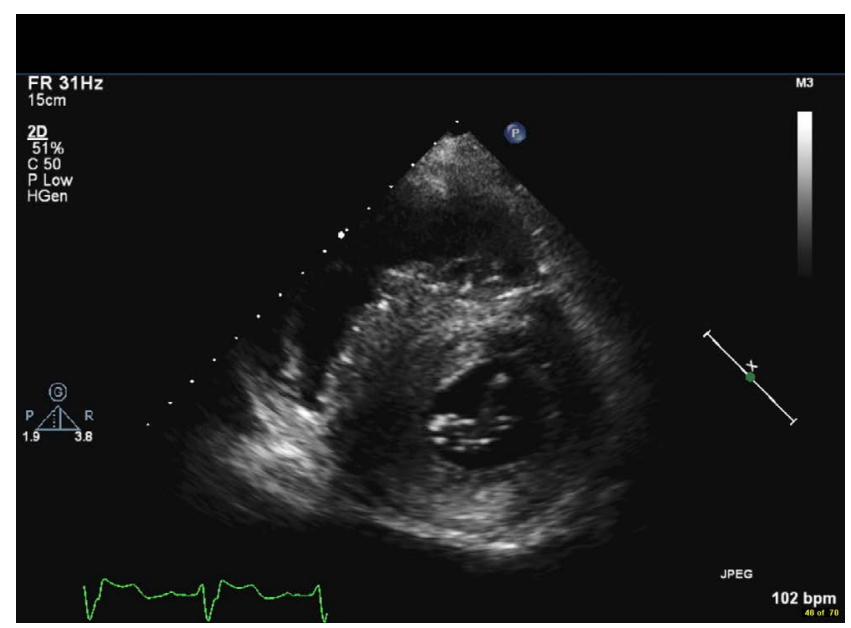

B

Figure 1: Still images of the transthoracic echocardiogram (TTE) obtained during initial evaluation at the Boston University Amyloidosis Center. A) Parasternal long-axis; B) and parasternal short-axis views reveal severe asymmetric septal hypertrophy and a left-sided pleural effusion.

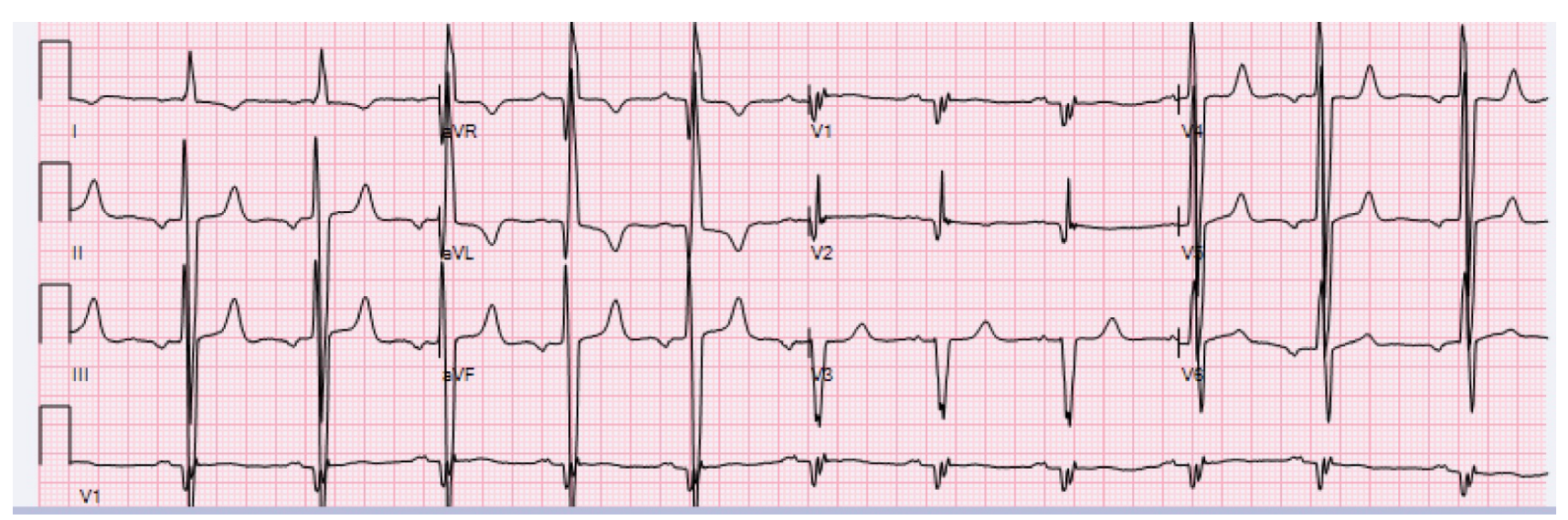

Figure 2: Routine ECG during Cardiology Clinic Visit. ECG reveals sinus rhythm, left ventricular hypertrophy, left anterior fascicular block, and ST-T waves changes consistent with repolarization abnormality. 
tion of serum free light concentration, but persistent monoclonal protein in serum and urine by immunofixation electrophoresis and 5-10\% lambda-restricted plasma cells on subsequent bone marrow biopsies.

A screening transthoracic echo at presentation to our center showed a severely increased interventricular septal thickness of $22 \mathrm{~mm}$, grade 1 diastolic dysfunction pattern, systolic anterior motion of the mitral valve with a left ventricular outflow gradient of $46 \mathrm{mmHg}$, mild mitral regurgitation, and left ventricular ejection fraction (LVEF) of $50 \%$ (Figure 1, Video $1 \mathrm{~A}$, Video $1 \mathrm{~B}$ and Video
1C). Further testing demonstrated a serum Brain Natriuretic Peptide of $1388 \mathrm{pg} / \mathrm{mL}$ (normal <53.2 pg/ml). An electrocardiogram showed normal sinus rhythm, left ventricular hypertrophy, left anterior fascicular block, and ST-T waves changes consistent with repolarization abnormality (Figure 2). Cardiac MRI (CMR) revealed a septal thickness of $19 \mathrm{~mm}$ and left ventricular mass of $532 \mathrm{~g}$, LVEF of $59 \%$, right ventricular ejection fraction of $52 \%$, and a single focus of delayed enhancement within the anterior wall in a pattern that did not suggest an infiltrative cardiomyopathy (Figure 3 and Video 2). These

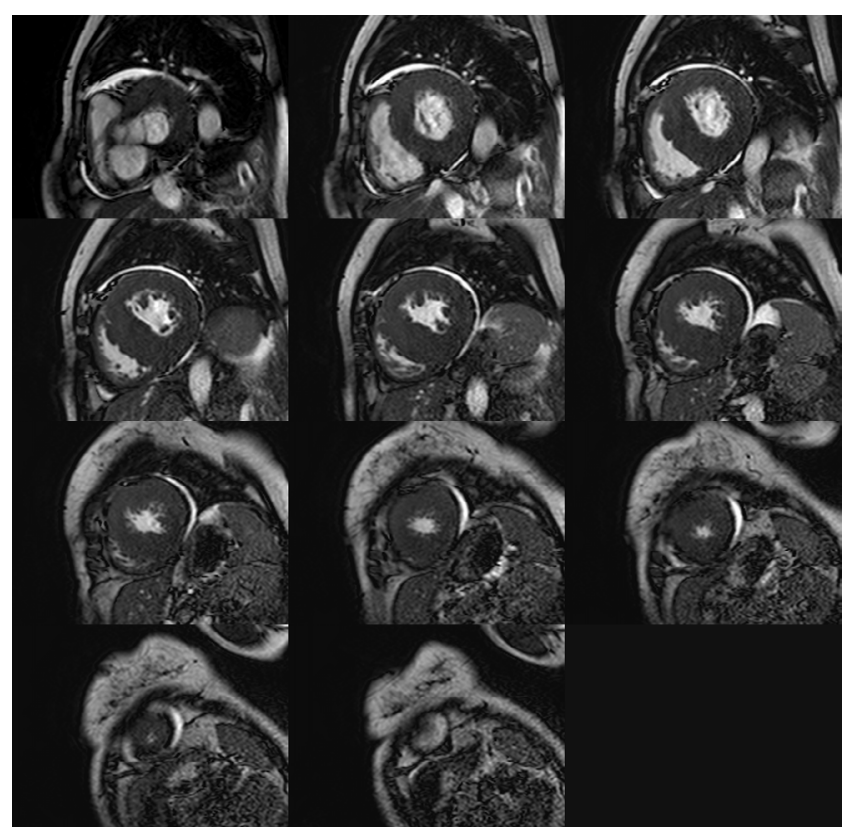

A

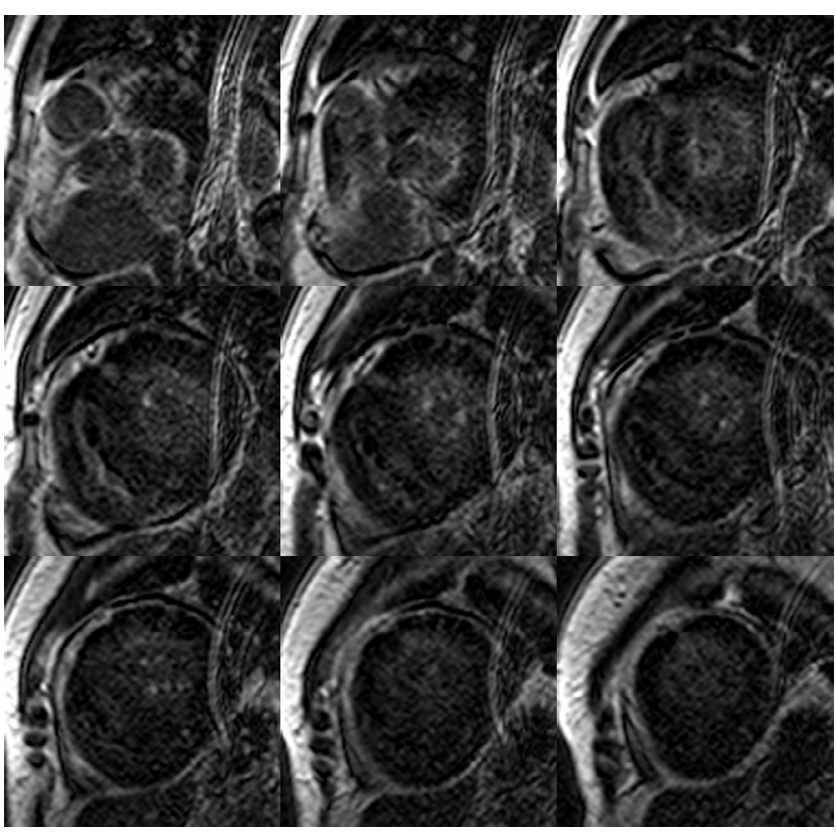

B

Figure 3: Cardiac MRI. A) Still-frame cine cardiac MRI contiguous short axis image slices from base to apex are illustrated. Note the prominent and asymmetric septal thickening consistent with HCM; B) Still frame late gadolinium enhancement (LGE) cardiac MRI short axis image slices from base to apex are illustrated. Note the focal late enhancement (single arrow) of the interventricular septum in a pattern consistent with HCM. Also note the poor contrast between myocardium and blood pool with subtle subdendocardial LGE (double arrow) that could also be consistent with cardiac amyloidosis.

Table 2: Clinical characteristics and laboratory data of the patient from diagnosis until death.

\begin{tabular}{|l|l|l|l|l|}
\hline Date & NYHA Class & BNP $(\mathbf{p g} / \mathrm{mL}) \mathbf{n m l ~ 0 - 7 2 . 3}$ & $\begin{array}{l}\text { Troponin I }(\mathbf{n g} / \mathbf{m L}): \text { Normal } \\
<\mathbf{0 . 0 1 3}\end{array}$ & $\begin{array}{l}\text { Orthostatic } \\
\text { Hypotension }\end{array}$ \\
\hline Initial visit $(\mathrm{t})$ & 1 & 1388 & & 0 \\
\hline $\mathrm{t}+2 \mathrm{yr}$ & 1 & 1133 & 0.343 & 0 \\
\hline $\mathrm{t}+5 \mathrm{yr}$ & III & 2012 & 0.033 & 0 \\
\hline
\end{tabular}

NYHA: New York Heart Association; BNP: Brain Natriuretic Peptide.

Table 3: Echocardiographic characteristics of the patient from diagnosis until death.

\begin{tabular}{|l|l|l|l|l|l|l|}
\hline Date & $\begin{array}{l}\text { LVEF } \\
\mathbf{( \% )}\end{array}$ & $\begin{array}{l}\text { Maximal } \\
\text { Interventricular Septal } \\
\text { Thickness }(\mathbf{m m})\end{array}$ & $\begin{array}{l}\text { Posterior Wall } \\
\text { Thickness } \mathbf{( m m})\end{array}$ & $\begin{array}{l}\text { LVOT Peak } \\
\text { Velocity } \mathbf{( m / s})\end{array}$ & $\begin{array}{l}\text { LVOT Peak } \\
\text { Gradient } \\
\mathbf{( m m H g )}\end{array}$ & $\begin{array}{l}\text { Degree of MR } \\
\mathbf{( 0 - 4 + )}\end{array}$ \\
\hline Initial visit (t) & 50 & 22 & 22 & 3.4 & 46 & $1+$ \\
\hline $\mathrm{t}+2 \mathrm{yr}$ & 40 & & & 5.4 & 114 & $2+$ \\
\hline $\mathrm{t}+5 \mathrm{yr}$ & 58 & 29 & 24 & $\begin{array}{l}\text { Could not } \\
\text { estimate }\end{array}$ & $\begin{array}{l}\text { Could not } \\
\text { estimate }\end{array}$ & $1+$ \\
\hline
\end{tabular}

LVEF: Left ventricular ejection fraction; LVOT: Left ventricular outflow tract; MR: Mitral regurgitation. 
CMR and echocardiographic findings confirmed a diagnosis of hypertrophic cardiomyopathy (HCM).

Blood testing for cardiac sarcomere mutations was sent and family members screened. There were no affected first-degree relatives, however the patient's genetic testing was significant for a missense mutation in $\mathrm{MYH7}$, a $\beta$-myosin heavy chain gene, associated with HCM [1].

Over a five-year period, the patient slowly started to develop signs and symptoms of congestive heart failure (Table 2). The LVEF fluctuated, but largely remained preserved (Table 3). However, the septal wall thickness gradually began to increase and reached up to $29 \mathrm{~mm}$.

Six years after her initial presentation, the patient was acutely hospitalized for decompensated heart failure and ultimately died of cardiogenic shock. A focused autopsy was performed, revealing cardiomegaly, left ventricular hypertrophy (990 grams), right ventricular hypertrophy $(10 \mathrm{~mm})$ and increased ventricular septal thickness (maximum thickness $30 \mathrm{~mm}$ ). There was extensive interstitial birefringent congophilic material in the left ventricle and interventricular septum, diagnostic of cardiac amyloidosis. The uninvolved myocardial fibers were hypertrophied with myocyte disarray, consistent with the diagnostic histological features of $\mathrm{HCM}$ (Figure 4 and Figure 5).

\section{Conclusion}

We present an unusual case of a woman with severely increased $L V$ wall thickness and systemic $A L$ amyloidosis who was ultimately diagnosed with both hypertrophic cardiomyopathy and AL cardiac amyloidosis at autopsy.

The systemic amyloidosis is a group of diseases caused by organ deposition of misfolded protein fragments. Disease manifestations and clinical course vary depending upon the precursor protein. In AL amyloido-

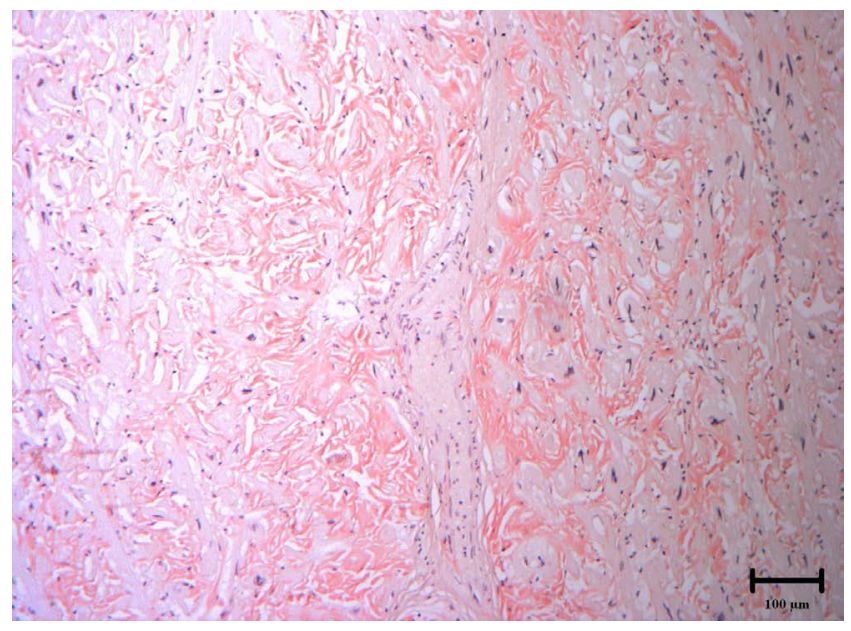

A

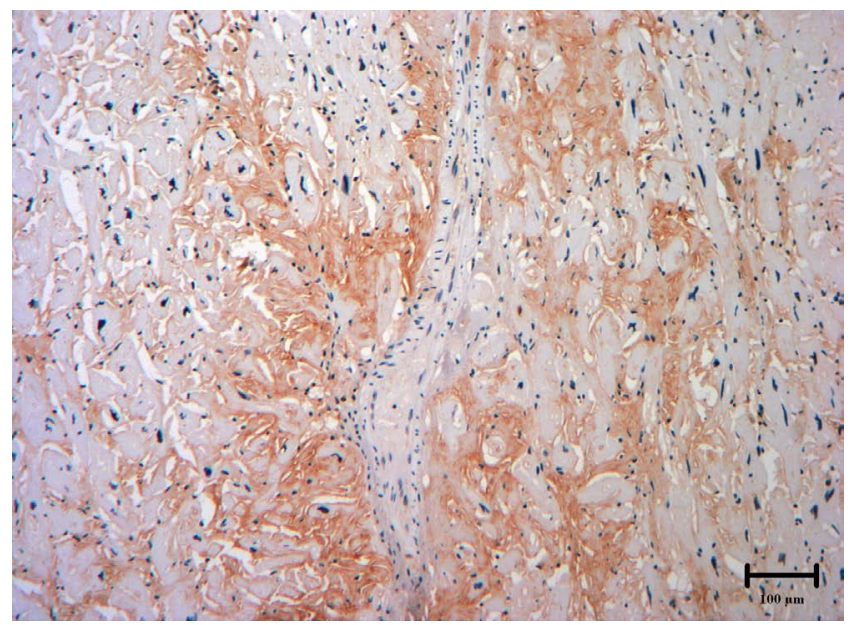

C

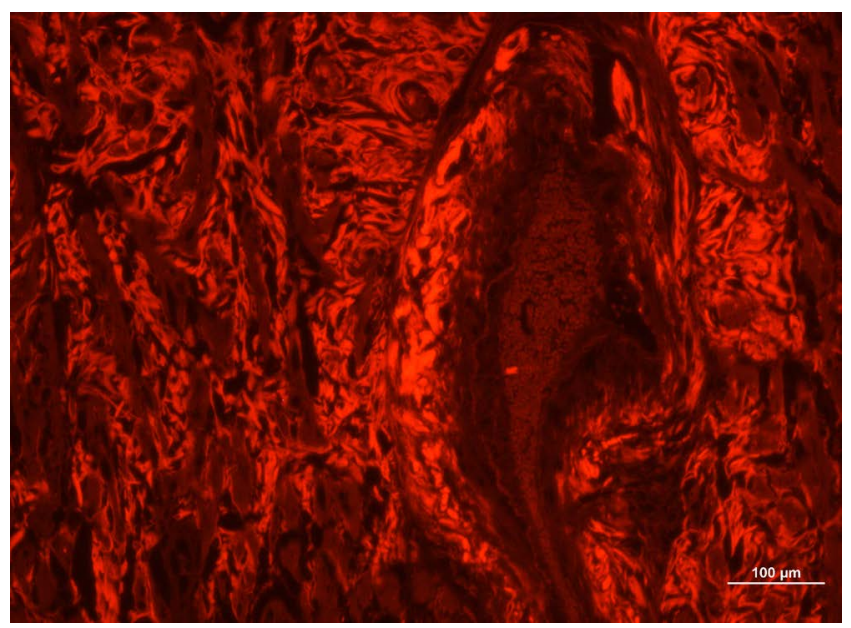

B

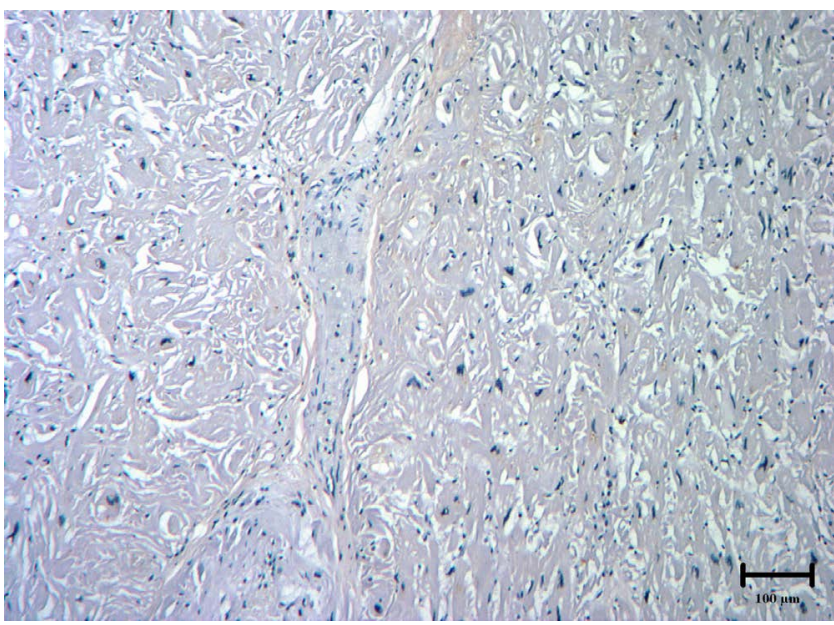

D

Figure 4: Cardiac histology samples from autopsy demonstrating extensive AL lambda amyloid deposition. Images are 100× magnification.

A) Extensive Congo red staining under white light, revealing extracellular amyloid fibril deposition; B) Congo red staining viewed under fluorescence light; C) Anti-lambda light chain antibody staining identifying lambda light chain fragments in the extracellular amyloid deposits, confirming the diagnosis of lambda AL cardiac amyloidosis; D) Anti-transthyretin light chain antibody reveals no significant transthyretin protein fragments, thus ruling out ATTR cardiac amyloidosis. 


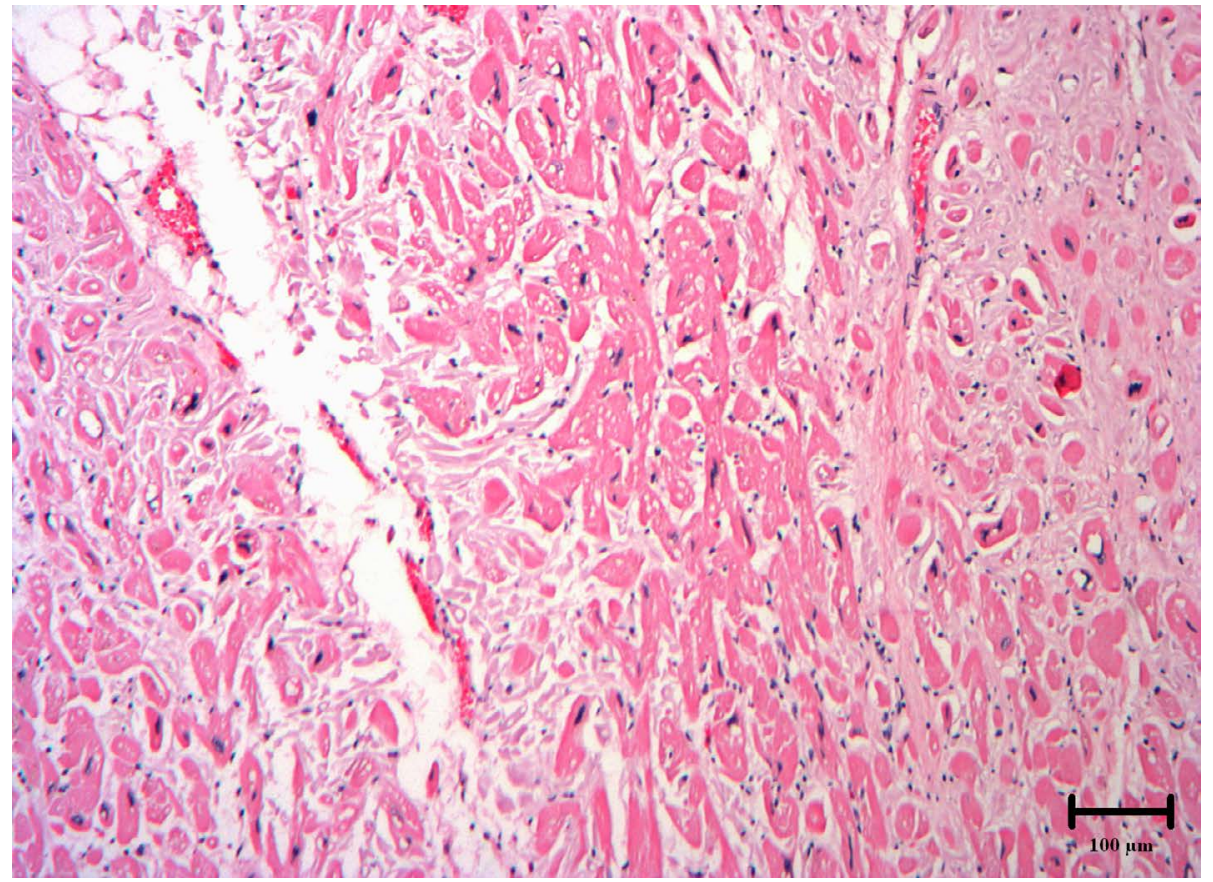

Figure 5: Autopsy heart section exhibiting the histology of hypertrophic cardiomyopathy. 100× magnification. H\&E staining reveals enlarged and disarrayed myocytes with hyperchromatic nuclei and intercellular fibrosis.

sis, the amyloidogenic protein is derived from monoclonal immunoglobulin light chains in the setting of a plasma cell dyscrasia. The incidence of AL amyloidosis is estimated to be about 9 cases per million people per year [2,3]. Tissue involvement with amyloid protein is diagnosed by light microscopy revealing characteristic apple-green birefringence with Congo red staining [4]. Cardiac involvement is common, affecting up to $50-70 \%$ of patients with systemic AL amyloidosis $[5,6]$. In contrast to AL amyloidosis, transthyretin (ATTR) cardiac amyloidosis is a distinct cause of cardiac amyloidosis which may be slowly progressive (particularly wild-type ATTR), and most resembles HCM in its echocardiographic appearance.

Cardiac amyloidosis is characterized by increased LV wall thickness, diastolic dysfunction, and low voltages on ECG [7]. The disease course in untreated patients is often one of rapidly progressive heart failure due to the underlying restrictive cardiomyopathy [8], often complicated by advanced conduction system disease and rarely, ventricular arrhythmias [9].

Hypertrophic cardiomyopathy (HCM) is a genetic cardiomyopathy caused by autosomal dominant mutations in genes encoding for the sarcomere and its associated myofibrils, with a prevalence of about 1 in 500 people worldwide [10]. While HCM is defined as LV hypertrophy (typically with septal wall thickness > $15 \mathrm{~mm}$ ), in the absence of other cardiac or systemic diseases leading to LV hypertrophy, overlap between HCM and other causes of LV hypertrophy has been well described. A prior report from our institution demonstrates the phenotypic overlap that can occur between cardiac amyloidosis and HCM, with four out of 97 new cases of AL amyloidosis displaying echocardiographic findings typically associated with HCM, including LV outflow gradients and mitral systolic anterior motion [11]. There are few reports, of both HCM and amyloid cardiomyopathy occurring in the same patient [12], and even fewer with histopathological confirmation. In a case series of septal myectomy for presumed HCM, three out of 204 patients were found to have amyloid deposits, and all were over the age of 60-years with wild-type ATTR as the precursor protein [13].

On imaging, cardiac amyloidosis can be distinguished from HCM by traditional echo parameters, including more advanced LV diastolic dysfunction and low normal or impaired LV systolic function. There is considerable overlap in echocardiographic features, however, and these and other classic parameters lack sensitivity in distinguishing cardiac amyloidosis from HCM [14]. Recently, the inclusion of various cardiac deformation parameters increases the sensitivity and specificity for the detection of amyloidosis, especially the LVEF to global longitudinal strain ratio [14]. In addition, a non-invasive, diagnostic algorithm can accurately be used to diagnosis cardiac ATTR amyloidosis, even in cohorts with HCM [15].

Our patient had markedly increased ventricular wall thickness consistent with either cardiac amyloidosis or HCM. The patient had autopsy-proven, extensive extracellular amyloid deposition in the heart in the setting of known AL amyloidosis, consistent with a diagnosis of cardiac amyloidosis [16]. In addition to her cardiac amyloidosis, she was genotype and phenotype positive for HCM. The preponderance of clinical evidence suggested concomitant HCM as well: Asymmetric septal hyper- 
trophy, systolic anterior motion of the mitral valve, and an autopsy revealing myocyte disarray characteristic of HCM in areas unaffected by amyloid deposition.

At initial presentation, this patient likely had HCM alone, given the significant interventricular septal thickness and minimal diastolic dysfunction. CMR supported this diagnosis, as there was only a single focus of delayed gadolinium enhancement within the anterior wall, as opposed to the diffuse delayed gadolinium enhancement that classically present in amyloid cardiomyopathy [16]. In patients with known systemic amyloidosis, CMR with late gadolinium enhancement has $86 \%$ sensitivity and $67 \%$ negative predictive value in diagnosis cardiac involvement [9]. The initial diagnosis of HCM was also supported by the fact that the patient maintained an excellent functional status for years, which was followed by the rapidly progressive clinical decline that characterizes AL cardiac amyloidosis. This clinical trajectory has also been described recently in another report of HCM and cardiac amyloidosis coexisting in the same patient [12].

This case highlights the importance of tissue diagnosis and genetic analysis in the evaluation of LV wall thickening when conflicting clinical features suggest divergent diagnoses. It also demonstrates the rapid clinical deterioration that can occur once a patient develops the superimposition of AL amyloid cardiomyopathy.

There was no support to disclosure: National Institutes of Health (NIH); Wellcome Trust; and other(s).

All authors precipitated in analysis and interpretation of the data, drafting the manuscript, acquiring key figures, and/or critical revisions for important intellectual content.

\section{References}

1. Richard P, Charron P, Carrier L, Ledeuil C, Cheav T, et al (2003) Hypertrophic Cardiomyopathy. Circulation 107.

2. Kyle RA, Gertz MA (1995) Primary systemic amyloidosis: Clinical and laboratory features in 474 cases. Semin Hematol 32: 45-59.

3. Sipe JD, Cohen AS (2000) Review: History of the amyloid fibril. J Struct Biol 130: 88-98.

4. Seldin DC, Berk JL, Sam F, Sanchorawala V (2011) Amyloidotic cardiomyopathy: Multidisciplinary approach to diagnosis and treatment. Heart Fail Clin 7: 385-393.
5. Ruberg FL, Berk JL (2012) Transthyretin (TTR) cardiac amyloidosis. Circulation 126: 1286-1300.

6. Gertz MA, Comenzo R, Falk RH, Fermand JP, Hazenberg BP, et al. (2004) Definition of organ involvement and treatment response in immunoglobulin light chain amyloidosis $(A L)$ : A consensus opinion from the 10th International Symposium On Amyloid And Amyloidosis, Tours, France, 18-22 April 2004. Am J Hematol 79: 319328.

7. Sanchorawala V (2006) Light-chain (AL) amyloidosis: Diagnosis and treatment. Clin J Am Soc Nephrol 1: 13311341.

8. Ruberg FL, Appelbaum E, Davidoff R, Ozonoff A, Kissinger $\mathrm{KV}$, et al. (2009) Diagnostic and prognostic utility of cardiovascular magnetic resonance imaging in light-chain cardiac amyloidosis. Am J Cardiol 103: 544-549.

9. Gersh BJ, Maron BJ, Bonow RO, Dearani JA, Fifer MA, et al. (2011) 2011 ACCF/AHA Guideline for the diagnosis and treatment of hypertrophic cardiomyopathy: A report of the American College of Cardiology Foundation/American Heart Association Task Force on Practice Guidelines. Developed in collaboration with the American Association for Thoracic Surgery, American Society of Echocardiography, American Society of Nuclear Cardiology, Heart Failure Society of America, Heart Rhythm Society, Society for Cardiovascular Angiography and Interventions, and Society of Thoracic Surgeons. J Am Coll Cardiol 58: e212-e260.

10. Dinwoodey DL, Skinner M, Maron MS, Davidoff R, Ruberg FL (2008) Light-chain amyloidosis with echocardiographic features of hypertrophic cardiomyopathy. Am J Cardiol 101: 674-676.

11. Tomberli B, Cappelli F, Perfetto F, Olivotto I (2017) Abrupt onset of refractory heart failure associated with light-chain amyloidosis in hypertrophic cardiomyopathy. JAMA Cardiol 2: 94.

12. Lamke GT, Allen RD, Edwards WD, Tazelaar HD, Danielson GK (2003) Surgical pathology of subaortic septal myectomy associated with hypertrophic cardiomyopathy. A study of 204 cases (1996-2000). Cardiovasc Pathol 12: 149-158.

13. Pagourelias ED, Mirea O, Duchenne J, Van Cleemput J, Delforge M, et al. (2017) Echo parameters for differential diagnosis in cardiac amyloidosis: A head-to-head comparison of deformation and nondeformation parameters. Circ Cardiovasc Imaging 10: e005588.

14. Gillmore JD, Maurer MS, Falk RH, Merlini G, Damy T, et al. (2016) Nonbiopsy diagnosis of cardiac transthyretin amyloidosis. Circulation 133: 2404-2412.

15. Falk RH (2005) Diagnosis and management of the cardiac amyloidoses. Circulation 112: 2047-2060.

16. Maceira AM, Joshi J, Prasad SK, Moon JC, Perugini E, et al. (2005) Cardiovascular magnetic resonance in cardiac amyloidosis. Circulation 111: 186-193. 